\title{
On Games of Perfect Information: Equilibria, $\varepsilon-$ Equilibria and Approximation by Simple Games*
}

\author{
Guilherme Carmona \\ Universidade Nova de Lisboa
}

February 26, 2003

\begin{abstract}
We show that every bounded, continuous at infinity game of perfect information has an $\varepsilon$-perfect equilibrium. Our method consists of approximating the payoff function of each player by a sequence of simple functions, and to consider the corresponding sequence of games, each differing form the original game only on the payoff function. In addition, this approach yields a new characterization of perfect equilibria: a strategy $f$ is a perfect equilibrium in such a game $G$ if and only if it is an $1 / n$-perfect equilibrium in $G_{n}$ for all $n$, where $\left\{G_{n}\right\}$ stand for our approximation sequence.
\end{abstract}

\section{Introduction}

Games of perfect information provide a useful framework in order to understand some economic problems. In such games, the notion of perfect equilibrium is the natural equilibrium concept since it captures the notion of sequential rationality: in particular, because players can compute opponents' optimal future behavior at each point in time, they will not be misled

\footnotetext{
*I wish to thank Kemal Badur, and Jan Werner for very helpful comments. Financial support from the Subprograma Ciência e Tecnologia do $2^{\circ}$ Quadro Comunitário de Apoio is gratefully acknowledged.
} 
by empty threats made by their opponents. Therefore, the question of existence of perfect equilibrium in games of perfect information is clearly of interest.

A general existence theorem was established by Harris [6]. His result applies to games of perfect information in which players' payoff functions are continuous, and their choice sets are compact sets that depend continuously on the choices made previously. In this note we show that any game of perfect information has an $\varepsilon$-perfect equilibrium, assuming only that players' payoff functions are bounded, and continuous at infinity. In this way, we presents a method for establishing existence of perfect equilibria in bounded, continuous at infinity games of perfect information: we need only to check whether the particular game we are interested in satisfies enough continuity and compactness properties to guarantee that limits of sequences of outcomes induced by $\varepsilon$-perfect equilibria, with $\varepsilon$ converging to zero, are perfect equilibrium outcomes. ${ }^{1}$

Our approach consists of approximating the payoff function of each player by a sequence of simple functions, in a way that is standard in measure and integration theory (see, for example, Wheeden and Zygmund [10]). The sequence of approximating payoff functions for each player, in addition of being a sequence of simple functions, satisfies two other important properties: first, it converges uniformly to the payoff function of that player in the original game; and second, any approximating function depends only on finite histories in the following sense: we can find an integer $t$ such that if any two infinite histories coincide on the first $t$ periods then they yield the same payoff.

The above approximation of each player's payoff function induces a sequence of games that differ from the original game only on the payoff function. For this sequence of games, it is easy to establish that each of them has a perfect equilibrium since they can be solved by backwards induction. Also, given $\varepsilon>0$, perfect equilibria of the approximating games far out in the sequence will be $\varepsilon$-perfect equilibria of the original game. Thus, every bounded, continuous at infinity game of perfect information has a $\varepsilon$-perfect equilibrium, for all $\varepsilon>0$. Furthermore, we can find a subsequence of the approximating games in a way that the set of perfect equilibria of the original game $G$ consists of those strategies that, for all $n$, are $1 / n$-perfect equilibria

\footnotetext{
${ }^{1}$ That it is enough to focus on outcomes rather than on strategies follows from Börgers [2], and Hellwig et al. [8].
} 
of $G_{n}$, where $\left\{G_{n}\right\}$ denotes the sequence of approximating games.

Our work is, thus, related to that of Fundenberg and Levine [3], and [4], Harris [5], and Börgers [1], all of which present characterizations of perfect equilibria in terms of $\varepsilon$-equilibria of truncated games. Our results are also related to the limiting results of Börgers [2] and Hellwig et al. [8]. Our approach differs from theirs essentially on the approximations to a given game that we consider. Given a game $G$ of perfect information, we consider approximating games that differ from $G$ only on the payoff functions; in addition, the sequence of payoff functions of the approximation games converges uniformly to the payoff functions of $G$. These two properties make it very easy to deal with limiting properties.

\section{Games of Perfect Information}

Our notation follows closely the one used by Harris [6]. There is a finite set of players $1 \leq i \leq N$, each of which chooses independently some action in every period of time, time being discrete. For $t \in \mathbb{N}$, period $t$ 's outcome is an element of $S_{t}=\times_{i=1}^{N} S_{t i}$. An history of the game is an element of $S=\{0\} \times \times_{t=1}^{\infty} S_{t}$, where $\{0\}$ is included for notational convenience. Given $x=\left(x_{0}, x_{1}, \ldots\right) \in S$, and $t \in \mathbb{N}, \lambda_{t} x=\left(x_{0}, \ldots, x_{t}\right)$ denotes the first $t$ components of $x$, and $\mu_{t} x=\left(x_{t}, x_{t+1}, \ldots\right)$ the sequence of outcomes from $t$ onwards.

A game of perfect information is a pair $G=(H, P)$ where $H$ is a nonempty subset of $S$, and $P: H \rightarrow \mathbb{R}^{N}$ (the function $P_{i}: H \rightarrow \mathbb{R}$ is player $i$ 's payoff function), satisfying the property that at each point in time only one player can make a choice. Formally, for any $t \in \mathbb{N}$, and $x \in H$ let $A_{t}\left(\lambda_{t-1} x\right)=\left\{y_{t}: y \in H, \lambda_{t-1} y=\lambda_{t-1} x\right\}$ denote the set of outcomes that are possible $\mathrm{n}$ period $t$ given the history $\lambda_{t-1} x$. As players choose independently, then $A_{t}\left(\lambda_{t-1} x\right)=\times_{i=1}^{N} A_{t i}\left(\lambda_{t-1} x\right)$. The assumption that at each point in time only one player can make a choice is formalized by imposing that for all $t \in \mathbb{N}$, and $x \in H$, at most one of the sets $\left\{A_{t i}\left(\lambda_{t-1} x\right)\right\}$ is not a singleton.

A strategy for player $i, 1 \leq i \leq N$, is a sequence of functions $f_{i}=\left\{f_{t i}\right\}_{t=1}^{\infty}$, where $f_{t i}: \lambda_{t-1} H \rightarrow S_{t i}$, and $f_{t i}\left(\lambda_{t-1} x\right) \in A_{t i}\left(\lambda_{t-1} x\right)$, for all $t \in \mathbb{N}$, and $x \in$ $H$. A strategy profile $f$ is a vector of individual strategies: $f=\left(f_{1}, \ldots, f_{N}\right)$. Let $F(H)$ denote the set of all strategy profiles (or strategies, for short), and $F^{i}(H)$ denote the set of all player $i$ 's strategies. If $h^{i} \in F^{i}(H)$, and $f \in F(H)$, then $f \backslash h^{i}$ denotes the strategy resulting from replacing player 
$i$ 's strategy $f^{i}$ by $h^{i}$.

Given a strategy $f, x \in H$, and $t \in \mathbb{N} \alpha[f, x, t] \in H$ denotes the history resulting if $f$ is employed from period $t+1$ onwards after an initial history $\lambda_{t} x$. For $\varepsilon \geq 0$, a strategy $f$ is an $\varepsilon$-perfect equilibrium of the game $G=(H, P)$ if $P_{i}(\alpha[f, x, t]) \geq P_{i}\left(\alpha\left[f \backslash h^{i}, x, t\right]\right)-\varepsilon$ for all $1 \leq i \leq N, h^{i} \in F^{i}(H), x \in H$, and $t \geq 0$. A strategy $f$ is a perfect equilibrium of the game $G=(H, P)$ if it is a 0 -perfect equilibrium. Let $P E(G)$ denote the set of perfect equilibria of the game $G$, and $P E_{\varepsilon}(G)$ the set of $\varepsilon$-perfect equilibria of the game $G$.

A game $(H, P)$ of perfect information is continuous at infinity if for all $\varepsilon>0$ and all $1 \leq i \leq N$, there exists $T \in \mathbb{N}$, such that $\left|P_{i}(x)-P_{i}(y)\right|<\varepsilon$ for all $x, y \in H$ satisfying $\lambda_{T}(x)=\lambda_{T}(y)$. This definition is due to Fudenberg and Levine [3]. A game $(H, P)$ of perfect information is bounded if for all $1 \leq i \leq N, P_{i}$ is bounded.

\section{Approximation by Simple Games, Existence of Perfect Equilibria and of $\varepsilon-$ Perfect Equi- libria}

In this section we show that every bounded, and continuous at infinity game has an $\varepsilon$-perfect equilibrium by using simple games approximations. We then use the model of Harris and Vickers [7] to show how this fact can be used to prove existence of perfect equilibria. Also, we characterize the set of perfect equilibria of any bounded, and continuous at infinity game in terms of $\varepsilon$-perfect equilibrium of those simple games.

Theorem 1 Let $G=(H, P)$ be a game of perfect information, and assume that $G$ is continuous at infinity, and bounded. Then, for all $\varepsilon>0, G$ has an $\varepsilon$-perfect equilibrium.

In order to prove Theorem 1, we will use the following construction: By Wheeden and Zygmund [10, theorem 4.13, page 54] and the remark immediately after, for all $1 \leq i \leq N$ there exist a sequence $\left\{V_{i}^{k}\right\}_{k}$ of simple functions converging uniformly to $P_{i}$. If $B>0$ is such that $\left|P_{i}(x)\right|<B$, then, for $k \in \mathbb{N}$, we may let $V_{i}^{k}: H \rightarrow \mathbb{R}$ be defined by

$$
P_{i}^{k}(h)=\frac{j-1}{2^{k}} \quad \text { if } \quad \frac{j-1}{2^{k}} \leq P_{i}(h)<\frac{j}{2^{k}}
$$


for $j=-B 2^{k}, \ldots, B 2^{k}$. Define $P_{i}^{k}: H \rightarrow \mathbb{R}$ by

$$
P_{i}^{k}(x)=\inf \left\{V_{i}^{k}(y): \lambda_{k} y=\lambda_{k} x\right\},
$$

and define $G_{k}=\left(H, P_{k}\right)$. The sequence $\left\{G_{k}\right\}$ is a sequence of simple games that approximate $G$ in the following sense.

Lemma 1 Let $G=(H, P)$ be a game of perfect information, and assume that $G$ is continuous at infinity, and bounded. Let $G_{k}$ be as above. Then,

1. $\lim _{k \rightarrow \infty}\left\|V_{i}^{k}-P_{i}^{k}\right\|_{\infty}=0$,

2. $\lim _{k \rightarrow \infty}\left\|P_{i}^{k}-P_{i}\right\|_{\infty}=0$.

Proof. Let $\eta>0,1 \leq i \leq N$ and let $T \in \mathbb{N}$ be such that $k \geq T$ implies

$$
\left\|V_{i}^{k}-P_{i}\right\|_{\infty}<\eta
$$

and

$$
\left|P_{i}(x)-P_{i}(y)\right|<\eta
$$

for all $x, y \in H$ satisfying $\lambda_{T} x=\lambda_{T} y$.

Let $x \in H$ and $k \geq T$. If $y \in H$ is such that $\lambda_{k} y=\lambda_{k} x$, then $\lambda_{T} y=\lambda_{T} x$ and so

$\left|V_{i}^{k}(x)-V_{i}^{k}(y)\right| \leq\left|V_{i}^{k}(x)-P_{t}(x)\right|+\left|P_{i}(x)-P_{i}(y)\right|+\left|P_{i}(y)-V_{i}^{k}(y)\right|<3 \eta$,

which implies

$$
V_{i}^{k}(x)-3 \eta \leq P_{i}^{k}(x) \leq V_{i}^{k}(x)
$$

Hence,

$$
\left\|V_{i}^{k}-P_{i}^{k}\right\|_{\infty} \leq 3 \eta
$$

This establishes 1. Also, it implies that

$$
\left\|P_{i}^{k}-P_{i}\right\|_{\infty}<4 \eta
$$

which establishes that $P_{i}^{k}$ converges uniformly to $P_{i}$.

Note that the game $G_{k}$ has a perfect equilibrium. This follows because each player $i$ is indifferent between every strategy that prescribes the same actions in periods 1 through $k$. Hence, for $t \geq k+1$, we may specify some arbitrary action for the player choosing in period $t$ independently of the history and use backward induction to specify strategies for players choosing in periods 1 through $k$. This fact is established in the following lemma. 
Lemma 2 For all $k \in \mathbb{N}, G_{k}$ has a perfect equilibrium.

Proof. Let $1 \leq i \leq N, t \in \mathbb{N}, t>k$, and $x \in H$. Let $a \in A_{t i}\left(\lambda_{t-1} x\right)$ define $f_{t i}\left(\lambda_{t-1} x\right)=a$. This defines $\left\{f_{t}\right\}_{t=k+1}^{\infty}$. Clearly, $P_{i}^{k}(\alpha[f, x, t]) \geq$ $P_{i}^{k}\left(\alpha\left[f \backslash h^{i}, x, t\right]\right)$, for all $h^{i} \in F^{i}(H)$, since, by definition, we have that $P_{i}^{k}(x)=P_{i}^{k}(y)$ whenever $\lambda_{k} x=\lambda_{k} y$.

Consider $t \leq k$, and assume that $\left\{f_{j}\right\}_{j=t+1}^{\infty}$ has been already defined in a way that $P_{i}^{k}(\alpha[f, x, j]) \geq P_{i}^{k}\left(\alpha\left[f \backslash h^{i}, x, j\right]\right)$, for all $h^{i} \in F^{i}(H)$, and $j \geq t$. We have that $\left\{P_{i}^{k}\left(\alpha\left[f,\left(\lambda_{t-1} x, a\right), t\right]\right): a \in A_{t i}\left(\lambda_{t-1} x\right)\right\}$ is finite, since $P_{i}^{k}$ is simple. So there exists $a^{*}$ that maximizes $a \mapsto P_{i}^{k}\left(\alpha\left[f,\left(\lambda_{t-1} x, a\right), t\right]\right)$ in $A_{t i}\left(\lambda_{t-1} x\right)$. Thus, define $f_{t i}\left(\lambda_{t-1} x\right)=a^{*}$. In order to establish the inductive hypothesis for $t$, let $h^{i} \in F^{i}(H)$. Define $g^{i} \in F^{i}(H)$ by

$$
g_{l i}= \begin{cases}h_{l i} & \text { if } l=t \\ f_{l i} & \text { otherwise. }\end{cases}
$$

Let $y_{t}=\left(h_{t i}\left(\lambda_{t} x\right), f_{t,-i}\left(\lambda_{t} x\right)\right)$, and let $x \backslash y_{t}$ denote the history that differ from $x$ only because $x_{t}$ was replaced by $y_{t}$. By the inductive hypothesis, we have that $P_{i}^{k}\left(\alpha\left[f, x \backslash y_{t}, t\right]\right) \geq P_{i}^{k}\left(\alpha\left[f \backslash h^{i}, x \backslash y_{t}, t\right]\right)$, which implies that $P_{i}^{k}(\alpha[f \backslash$ $\left.\left.g^{i}, x, t-1\right]\right) \geq P_{i}^{k}\left(\alpha\left[f \backslash h^{i}, x, t-1\right]\right)$. Then, by construction, $P_{i}^{k}(\alpha[f, x, t-1]) \geq$ $P_{i}^{k}\left(\alpha\left[f \backslash g^{i}, x, t-1\right]\right)$.

Thus we have defined a strategy $f$ with the property that $P_{i}^{k}(\alpha[f, x, t]) \geq$ $P_{i}^{k}\left(\alpha\left[f \backslash h^{i}, x, t\right]\right)$, for all $1 \leq i \leq N, h^{i} \in F^{i}(H), x \in H$, and $t \geq 0$. Clearly, $f$ is a perfect equilibrium of $G_{k}$.

By lemma 2, it remains to show that any perfect equilibrium of $G_{k}$ is an $\varepsilon$-perfect equilibrium of $G$ provided that $k$ is sufficiently large. This is shown in the following lemma.

Lemma 3 Let $\varepsilon>0$. Then there is $K \in \mathbb{N}$ such that $k \geq K$ and $f \in$ $P E\left(G_{k}\right)$ imply that $f$ is an $\varepsilon$-perfect equilibrium of $G$.

Proof. Let $\varepsilon>0$ and let $K \in \mathbb{N}$ be such that $\left\|P_{i}^{K}-P_{i}\right\|_{\infty}<\frac{\varepsilon}{2}$. Let $f$ be perfect equilibrium of $G_{K}$. We will show that $f$ is an $\varepsilon$-perfect equilibrium of $G$.

Let $1 \leq i \leq N, h^{i} \in F^{i}(H), x \in H$, and $t \geq 0$. Then, $P_{i}^{K}(\alpha[f, x, t]) \geq$ $P_{i}^{K}\left(\alpha\left[f \backslash h^{i}, x, t\right]\right)$ and so

$$
\begin{aligned}
& \frac{\varepsilon}{2}+P_{i}(\alpha[f, x, t])>P_{i}^{K}(\alpha[f, x, t]) \geq \\
& \geq P_{i}^{K}\left(\alpha\left[f \backslash h^{i}, x, t\right]\right)>P_{i}\left(\alpha\left[f \backslash h^{i}, x, t\right]\right)-\frac{\varepsilon}{2},
\end{aligned}
$$


that is, $P_{i}\left(\alpha\left[f \backslash h^{i}, x, t\right]\right)-P_{i}(\alpha[f, x, t]) \leq \varepsilon$.

Part of the interest of approximating a given game $G$ of perfect information by the sequence $\left\{G_{k}\right\}$ of games is that each $G_{k}$ can be solved by backwards induction. This is clearly an important property when one is interested in computing the equilibrium set of any game of perfect information. Thus, it is interesting to know whether the equilibrium set of any such game $G$ can be computed using the games in $\left\{G_{k}\right\}$. The following result answers this question.

Theorem 2 Let $G=(H, P)$ be a game of perfect information, and assume that $G$ is continuous at infinity, and bounded. Let $G_{k}$ be as above. Then, there exists a subsequence $\left\{G_{k_{n}}\right\}$ of $\left\{G_{k}\right\}$ such that

$$
P E(G)=\bigcap_{n=1}^{\infty} P E_{\frac{1}{n}}\left(G_{n}\right) .
$$

Proof. For $n \in \mathbb{N}$, let $k_{n}$ be such that $\left\|V_{i}^{k_{n}}-P_{i}^{k_{n}}\right\|_{\infty}<1 / 2 n$; its existence is guaranteed by lemma 1 .

Let $f \in \cap_{n=1}^{\infty} P E_{\frac{1}{n}}\left(G_{n}\right), 1 \leq i \leq N, h^{i} \in F^{i}(H), x \in H$, and $t \geq 0$. Then, $P_{i}^{k_{n}}(\alpha[f, x, t]) \geq P_{i}^{k_{n}}\left(\alpha\left[f \backslash h^{i}, x, t\right]\right)-1 / n$, for all $n$, and so, by lemma 1 , it follows that $P_{i}(\alpha[f, x, t]) \geq P_{i}\left(\alpha\left[f \backslash h^{i}, x, t\right]\right)$. Hence $f \in P E(G)$.

Let $f \in P E(G)$. It is straightforward to verify that $P_{i}(x) \geq P_{i}(y)$ implies that $V_{i}^{k}(x) \geq V_{i}^{k}(y)$ for all $1 \leq i \leq N, k \in \mathbb{N}$, and $x, y \in H$. This clearly implies that $f$ is a perfect equilibrium of $\left(H, V^{k}\right)$. Let $n \in \mathbb{N}, 1 \leq i \leq N, h^{i} \in$ $F^{i}(H), x \in H$, and $t \geq 0$. Then, $V_{i}^{k_{n}}(\alpha[f, x, t]) \geq V_{i}^{k_{n}}\left(\alpha\left[f \backslash h^{i}, x, t\right]\right)$ together with $\left\|V_{i}^{k_{n}}-P_{i}^{k_{n}}\right\|_{\infty}<1 / 2 n$ imply $P_{i}^{k_{n}}(\alpha[f, x, t]) \geq P_{i}^{k_{n}}\left(\alpha\left[f \backslash h^{i}, x, t\right]\right)-1 / n$. Hence, $f \in \cap_{n=1}^{\infty} P E_{\frac{1}{n}}\left(G_{n}\right)$.

An important implication of Theorem 1 is that in order to establish the existence of a perfect equilibrium in a game of perfect information we need just enough continuity to guarantee that limits of $\varepsilon$-equilibria, with $\varepsilon>0$ converging to zero, exist and are equilibria. Sufficient conditions for this result are given in Reny [9], which although states for normal form games can be easily adapted to games of perfect information. However, sometimes the above property can be easily established directly. The model of Harris and Vickers [7] provide such an example.

Harris and Vickers [7] have modelled a race: two players, $A$ and $B$, are in competition for a single indivisible prize, which has value $V_{A}$ for player $A$ and $V_{B}$ for player $B$. At the outset of the game, players $A$ and $B$ are 
respectively at distances $x_{0}$ and $y_{0}$ from the finishing line; progress towards the finishing line depends upon the bids made by each player, which are the actions they choose. Players bid alternatively, first $A$, then $B$, then $A$ again and so on, and the prize is awarded to the first player (if any) to reach the finishing line.

More precisely, the game is as follows: there are two players, $A$ and $B$, which choose alternatively, first $A$, then $B$, then $A$ again and so on. Each player chooses a point from $S=[0, M]$, for some $M \in \mathbb{R}_{++}$, independently of the previous history.

To define players' payoffs we proceed as follows: let $x_{0}, y_{0}, V_{A}, V_{B}>0$, $0<\rho_{A}, \rho_{B}<1$ and let $w_{A}, w_{B}$ be strictly increasing, continuous function from $S$ to $\mathbb{R}$, satisfying $w_{A}(0)=w_{B}(0)=0$. Let $\left\{a_{2 k-1}\right\}_{k=1}^{\infty}$ and $\left\{b_{2 k}\right\}_{k=1}^{\infty}$ denote the choices of player $A$ and $B$, respectively, and define, for $k \geq 1$,

$$
x_{2 k-1}=x_{2(k-1)-1}-w_{A}\left(a_{2 k-1}\right)
$$

and

$$
y_{2 k}=y_{2(k-1)}-w_{B}\left(b_{2 k}\right) .
$$

Let $S^{\infty}=\times_{n \in \mathbb{N}} S_{n}$, with $S_{n}=S$, for all $n \in \mathbb{N}$. For $s \in S^{\infty}$, let $\gamma(s)$ denote the smallest integer $n$ such that either $x_{n} \leq 0$ or $y_{n} \leq 0$. Finally, the payoff for player $A$ is defined by

$$
P_{A}(s)= \begin{cases}\rho_{A}^{k-1} V_{A}-\sum_{i=1}^{\infty} \rho_{A}^{2 i-1} s_{2 i-1} & \text { if } \gamma(s)=k<\infty \text { and } k \text { is odd } \\ -\sum_{i=1}^{\infty} \rho_{A}^{2 i-1} s_{2 i-1} & \text { otherwise. }\end{cases}
$$

and the payoff for player $B$ is defined by

$$
P_{B}(s)= \begin{cases}\rho_{B}^{k-1} V_{B}-\sum_{i=1}^{\infty} \rho_{B}^{2 i} s_{2 i} & \text { if } \gamma(s)=k<\infty \text { and } k \text { is even } \\ -\sum_{i=1}^{\infty} \rho_{B}^{2 i-1} s_{2 i-1} & \text { otherwise. }\end{cases}
$$

Let $G_{R}$ denote the above game. Clearly, all the assumptions of Theorem 1 of Harris [6] are satisfied, except the continuity of the payoff functions. However, they are bounded, and continuous at the infinity. We will show the existence of a perfect equilibrium by a limit argument involving $\varepsilon$-perfect equilibria. An important part of the argument consists of showing that $P_{i}$, $i=A, B$, is continuous at limit points of $\varepsilon_{n}$-perfect equilibrium outcomes of $G_{R}, \varepsilon_{n} \rightarrow 0$.

Lemma 4 Let $\left\{f_{n}\right\}_{n=1}^{\infty}$ be a sequence of $\varepsilon_{n}$-perfect equilibria of $G_{R}, \varepsilon_{n} \rightarrow 0$, $i \in\{A, B\}, z \in H$, and $k \in \mathbb{N}$. If $\left\{\alpha\left[f_{n}, z, k\right]\right\}_{n=1}^{\infty}$ is a converging sequence of outcomes, with $v=\lim _{n} \alpha\left[f_{n}, z, k\right]$, then $\lim _{n} P_{i}\left(\alpha\left[f_{n}, z, k\right]\right)=P_{i}(v)$. 
Proof. Let $z \in H$, and $k \in \mathbb{N}$.

Note first that we may assume that under $z$ no player wins the race before $k$ (i.e., $x_{l}[v], y_{l}[v]>0$, for all $l \leq k$ ), since in this case the result is obvious.

We will assume that the player that moves in period $k+1$ is player $A$, the case in which the player that moves in period $k+1$ is player $B$ is exactly symmetric), and we will consider two cases:

Case 1: Under $v$, player $A$ wins the race in period $l>k$.

Let $l$ be such that $x_{l}[v] \leq 0$ and $x_{\kappa}[v]>0$, for all $\kappa<l$. Since $w_{A}$ is continuous, it follows that there is $N_{1} \in \mathbb{N}$ such that $x_{\kappa}\left[f_{n}, z, k\right]>0$, and $y_{\kappa}\left[f_{n}, z, k\right]>0$, for all $\kappa<k$, if $n \geq N_{1}$. Also, since $0<\rho_{A}<1$, there is $N_{2}$ such that $n \geq N_{2}$ implies

$$
\rho_{A}^{k-1} V_{A}-\sum_{i=1}^{\infty} \rho_{A}^{2 i-1} s_{2 i-1}[v]>\rho_{A}^{j-1} V_{A}-\sum_{i=1}^{\infty} \rho_{A}^{2 i-1} s_{2 i-1}\left[f_{n}, z, k\right]+\varepsilon_{n}
$$

for all $j>l$. Since $f_{n}$ is a $\varepsilon_{n}$-perfect equilibrium, it follows that $x_{l}\left[f_{n}, z, k\right] \leq$ 0 . Hence, for all $n \geq \max \left\{N_{1}, N_{2}\right\}$,

$$
P_{A}\left(\alpha\left[f_{n}, z, k\right]\right)=\rho_{A}^{l-1} V_{A}-\sum_{i=1}^{\infty} \rho_{A}^{2 i-1} s_{2 i-1}\left[f_{n}, z, k\right],
$$

and so

$$
\lim P_{A}\left(\alpha\left[f_{n}, z, k\right]\right)=P_{A}(v) .
$$

Case 2: Under $v$, player $B$ wins the race.

By the argument above for case 1 , it follows that $B$ will win the race under $f_{n}$, for $n$ big enough. Thus,

$$
P_{A}\left(\alpha\left[f_{n}, z, k\right]\right)=-\sum_{i=1}^{\infty} \rho_{A}^{2 i-1} s_{2 i-1}\left[f_{n}, z, k\right] \rightarrow-\sum_{i=1}^{\infty} \rho_{A}^{2 i-1} s_{2 i-1}[v]=P_{A}(v) .
$$

Case 3: Under $v$, neither player wins the race.

Let $\delta>0$, and let $L$ be such that $l \geq L$ implies $\rho_{A}^{l-1} V_{A}<\frac{\delta}{2}$. Let $N$ be such that $n \geq N$ implies that $x_{L}\left[f_{n}, z, k\right]>0$ and

$$
\left|\sum_{i=1}^{\infty} \rho_{A}^{2 i-1} s_{2 i-1}\left[f_{n}, z, k\right]-\sum_{i=1}^{\infty} \rho_{A}^{2 i-1} s_{2 i-1}[v]\right|<\frac{\delta}{2} .
$$


Then, since $x_{L}\left[f_{n}, z, k\right]>0$, it follows that

$-\sum_{i=1}^{\infty} \rho_{A}^{2 i-1} s_{2 i-1}\left[f_{n}, z, k\right] \leq P_{A}\left(\alpha\left[f_{n}, z, k\right]\right) \leq \rho_{A}^{L-1} V_{A}-\sum_{i=1}^{\infty} \rho_{A}^{2 i-1} s_{2 i-1}\left[f_{n}, z, k\right]$,

and so

$$
\left|P_{A}\left(\alpha\left[f_{n}, z, k\right]\right)-P_{A}(v)\right|<\frac{\varepsilon}{2}+\rho_{A}^{L-1} V_{A}<\varepsilon .
$$

We have show that $\lim _{n} P_{A}\left(\alpha\left[f_{n}, z, k\right]\right)=P_{A}(v)$. The argument present so far can be also used to show that $\lim _{n} P_{B}\left(\alpha\left[f_{n}, z, k\right]\right)=P_{B}(v)$ in the following way: if neither player wins the race, then the same argument can be used for player $B$; if player $A$ wins the race under $z$ then $\lim _{n} P_{B}\left(\alpha\left[f_{n}, z, k\right]\right)=P_{B}(v)$ can be show in the same way we showed that $\lim _{n} P_{A}\left(\alpha\left[f_{n}, z, k\right]\right)=P_{A}(v)$ in case 2 .

A second important part of the argument showing existence of a perfect equilibrium through a limit argument involving $\varepsilon$-perfect equilibria consists in defining a strategy which is in some sense a limit of the sequence of $\varepsilon$-perfect equilibria.

Lemma 5 Let $\left\{f_{n}\right\}_{n=1}^{\infty}$ be a sequence of $\varepsilon_{n}$-perfect equilibria of $G_{R}, \varepsilon_{n} \rightarrow 0$, and $f$ a strategy satisfying the following properties: for all $x \in H$, and $t \in \mathbb{N}$,

1. there exists a subsequence $\left\{f_{n}\right\}_{n \in D\left[\lambda_{t} x\right]}$ such that $\lim _{n \rightarrow \infty, n \in D\left[\lambda_{t} x\right]} \alpha\left[f_{n}, x, t\right]=$ $\alpha[f, x, t]$, and

2. $D\left[\lambda_{t+1} x\right] \subseteq D\left[\lambda_{t} x\right]$.

Then $f$ is a perfect equilibrium of $G_{R}$.

Proof. Let $i \in\{A, B\}, x \in H$, and $t \in \mathbb{N}$ be given, and consider a single deviation in period $t+1$ after history $\lambda_{t} x$. For concreteness, assume that player $A$ chooses in period $t+1$, and that he chooses $a$ instead of $f\left(\lambda_{t} x\right)$. Denote by $h^{A}$ the resulting player $A$ 's strategy. Note that $\alpha\left[f_{n} \backslash\right.$ $\left.h^{A}, x, t\right]=\alpha\left[f_{n}, y, t+1\right]$, where $y_{t}=a$, and $y_{k}=x_{k}$ for all $k \neq t$, and so $\lambda_{t} x=\lambda_{t} y$. We have that $P_{A}\left(\alpha\left[f_{n}, x, t\right]\right) \geq P_{A}\left(\alpha\left[f_{n} \backslash h^{A}, x, t\right]\right)-\varepsilon_{n}$. Since $D\left[\lambda_{t+1} y\right] \subseteq D\left[\lambda_{t} y\right]=D\left[\lambda_{t} x\right]$, then letting $n \rightarrow \infty$, with $n \in D[y, t+1]$, we obtain $P_{A}(\alpha[f, x, t]) \geq P_{A}\left(\alpha\left[f \backslash h^{A}, x, t\right]\right)$.

By Theorem 1, we know that for any bounded, continuous at infinity game there exists a sequence of $\varepsilon_{n}$-perfect equilibria with $\varepsilon_{n} \rightarrow 0$. Furthermore, the construction in Börgers [2], and Hellwig et al. [8] shows that given any such sequence we can define $f$ in a way that properties 1 and 2 above holds since $H$ is compact in $G_{R}$. Thus, we obtain the following corollary. 
Corollary $1 G_{R}$ has a perfect equilibrium.

We note that lemmas 4 and 5 provide a general approach to the question of existence of perfect equilibria in games of perfect information: any bounded, continuous at infinity game of perfect information for which those lemmas hold, and for which we can construct strategies in the way described in lemma 5 , it will have at least one perfect equilibrium.

\section{References}

[1] Börgers, T., (1989), "Perfect Equilibrium Histories of Finite and Infinite Horizon Games," Journal of Economic Theory, 47, 218-227.

[2] Börgers, T., (1991), "Upper Hemicontinuity of the Correspondence of Subgame-Perfect Equilibrium Outcomes," Journal of Mathematical Economics, 20, 89-106.

[3] Fudenberg, D., and D. Levine, (1983), "Subgame-Perfect Equilibria of Finite and Infinite Horizon Games," Journal of Economic Theory, 31, 251-268.

[4] Fudenberg, D., and D. Levine, (1986), "Limit Games and Limit Equilibria," Journal of Economic Theory, 38, 261-279.

[5] Harris, C., (1985), "A Characterizarion of the Perfect Equilibria of Infinite Horizon Games," Journal of Economic Theory, 37, 99-125.

[6] Harris, C., (1985), "Existence and Characterization of Perfect Equilibrium in Games of Perfect Information," Econometrica, 53, 613-628.

[7] Harris, C. and J. Vickers, (1985), "Perfect Equilibrium in a Model of a Race," Review of Economic Studies, 52, 193-209.

[8] Hellwig, M., W. Leininger, P. Reny and A. Robson, (1990), "Subgame Perfect Equilibrium in Continuous Games of Perfect Information: An Elementary Approach to Existence and Approximation by Discrete Games," Journal of Economic Theory, 52, 406-422.

[9] Reny, P., (1999), "On the Existence of Pure and Mixed Strategy Nash Equilibria in Discontinuous Games," Econometrica, 67, 1029-1056. 
[10] Wheeden, R. and A. Zygmund, (1977), Measure and Integral: an Introduction to Real Analysis, New York: Dekker. 